\title{
THE STUDY OF THE PROPERTIES OF THE EXTENDED HIGGS BOSON SECTOR WITHIN hMSSM MODEL
}

\author{
T. V. Obikhod *, E. A. Petrenko \\ Institute for Nuclear Research National Academy of Sciences of Ukraine, 03028 Kyiv, Ukraine
}

(Received January 29, 2018)

\begin{abstract}
Using the latest experimental data, performed by ATLAS Collaboration and within the framework of the Minimal Supersymmetric Standard Model, we presented the calculations for cross sections times branching fractions, $\sigma \times B r$, as a functions of the CP-even, H, Higgs boson mass, CP-odd, A, Higgs boson mass and charged, $\mathrm{H}^{ \pm}$, Higgs boson mass. Using the restricted parameter set, received from the hMSSM+HDECAY and "low-tb-high" scenarios, with the help of the computer programs SOFTSUSY, Prospino and SusHi, we received the large values of $\sigma \times B r$ for A and $\mathrm{H}$ bosons at $\tan \beta=2$ for the planned $14 \mathrm{TeV}$ at the LHC and found the large $\sigma \times B r$ at $\tan \beta=30$ for charged Higgs boson. The obtained results are of experimental interest as they are connected with the experimental searches for new physics beyond the Standard Model at the LHC.
\end{abstract}

PACS: 11.25.-w, 12.60.Jv, 02.10.Ws

\section{INTRODUCTION}

The searches for supersymmetry (SUSY) are motivated by the solutions of the most important problems: the hierarchy problem, gauge coupling unification and dark matter problem [1]. Experimental searches for SUSY in the most probable channels for the superparticle production at the LHC did not lead to the desired results and set new lower limits in the mass range about $2 \mathrm{TeV}$ for gluino and squarks [2]. This fact led to the need for SUSY searches in other sectors, for example, in the electroweak sector. As highlighted in CERN Courier [3]: "Based on data recorded in 2016, CMS has covered models of electroweak production of "wino"-like charginos and neutralinos with searches in different final states.

More results are expected soon, and the sensitivity of the searches will largely profit from the extension of the data set in the remaining two years of LHC Run 2". Another important sector for SUSY searches in low mass range of $1 \mathrm{TeV}$ are the searches for extended Higgs boson sector predicted by Minimal Supersymmetric Standard Model (MSSM) [4], that consists of five Higgs bosons: $\mathrm{CP}$ even Higgs bosons, h and H, CP odd Higgs boson, A, charged Higgs bosons, $\mathrm{H}^{ \pm}$.

The purpose of our paper is to calculate the production cross section of such particles at the energy of $14 \mathrm{TeV}$ at the LHC in the most optimal space of parameters of the MSSM model.

\section{OPTIMAL PARAMETER SPACE FOR STUDYING OF THE PROPERTIES OF MSSM HIGGS BOSONS}

The masses of five Higgs bosons of MSSM model at tree level are calculated through the masses of gauge boson, $\mathrm{M}_{W}, \mathrm{M}_{Z}$, and two additional parameters such as the pseudoscalar mass, $\mathrm{M}_{A}$ and the ratio of vacuum expectation values of two Higgs doublets, $\tan \beta \equiv v_{u} / v_{d}[5]$ :

$$
\begin{gathered}
M_{H^{ \pm}}^{2}=M_{A}^{2}+M_{W}^{2}, \\
M_{h, H}^{2}=\frac{1}{2}\left(M_{A}^{2}+M_{Z}^{2} \mp \sqrt{\left(M_{A}^{2}+M_{Z}^{2}\right)^{2}-4 M_{A}^{2} M_{Z}^{2} \cos ^{2} \beta}\right) .
\end{gathered}
$$

In the paper [6] the theoretical predictions of the MSSM Higgs particles in the low $\tan \beta$ regime, $1 \leq \tan \beta \leq 3$ are reviewed, with the assumption that SUSY should be in the range of $1 \mathrm{TeV}$. It was showed that the heavier MSSM neutral H/A and charged $\mathrm{H}^{ \pm}$states can decay into gauge bosons, lighter Higgs bosons and top quarks, presented in Fig.1.

In the Handbook of LHC Higgs cross sections, 2017 [5] are given examples of sensitivity on the $\left[\tan \beta, \mathrm{M}_{A}\right]$ parameter space for the "model independent" hMSSM approach [6], compared to the second approach [7] so called "low-tb-high" approach in the MSSM, that is orthogonal to the one previous. Relative differences in $\mathrm{BR}(\mathrm{H} \rightarrow \mathrm{WW})$ between the predictions of the "low-tb-high" scenario and the corresponding predictions obtained with the hMSSM+HDECAY combination are presented in Fig.2.

${ }^{*}$ Corresponding author E-mail address: obikhod@kinr.kiev.ua 

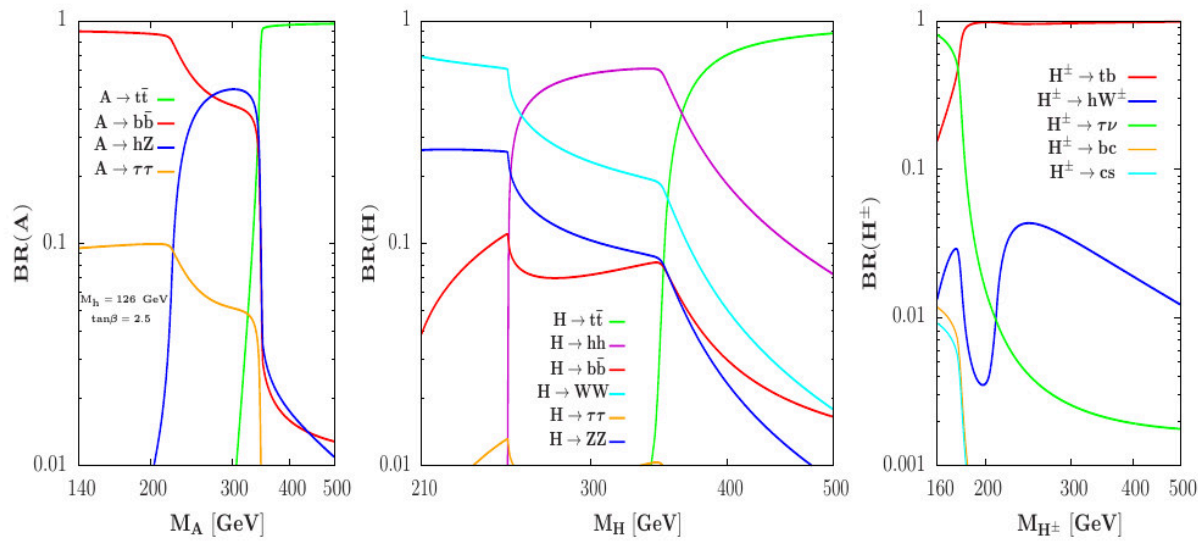

Fig.1. The branching ratios as functions of masses of MSSM Higgs bosons (A left, $H$ center, $H^{ \pm}$right) for $\tan \beta=2.5$, from $[6]$

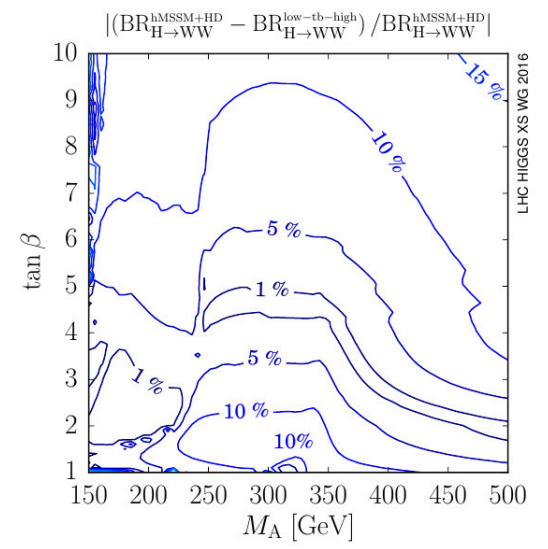

The results of ATLAS [8] and CMS [9] Collaborations excluded at the $95 \%$ confidence level (CL) a significant part of the $\left[\tan \beta, \mathrm{M}_{A}\right]$ plane. We'll use the benchmark scenarios of the model independent approach for the Higgs sector, the hMSSM with $\mathrm{M}_{h}=125 \mathrm{GeV}$ for the experimental limits on the cross sections times branching ratios in the context of the MSSM [10]. The results for the branching fractions received with the program HDECAY [11] for the Higgs decays in the $\left[\tan \beta, \mathrm{M}_{A}\right]$ plane are displayed in Fig. 3 with red area for the large decay rates and blue area for the small one.

The production cross sections for $\mathrm{A}$ and $\mathrm{H}$ bosons are displayed in Fig. 4 in the $\left[\tan \beta, \mathrm{M}_{A}\right]$ hMSSM pa-

Fig.2. Relative differences in $B R(H \rightarrow W W)$ between the hMSSM+HDECAY scenario and the "low-tb-high" scenario, from [5]
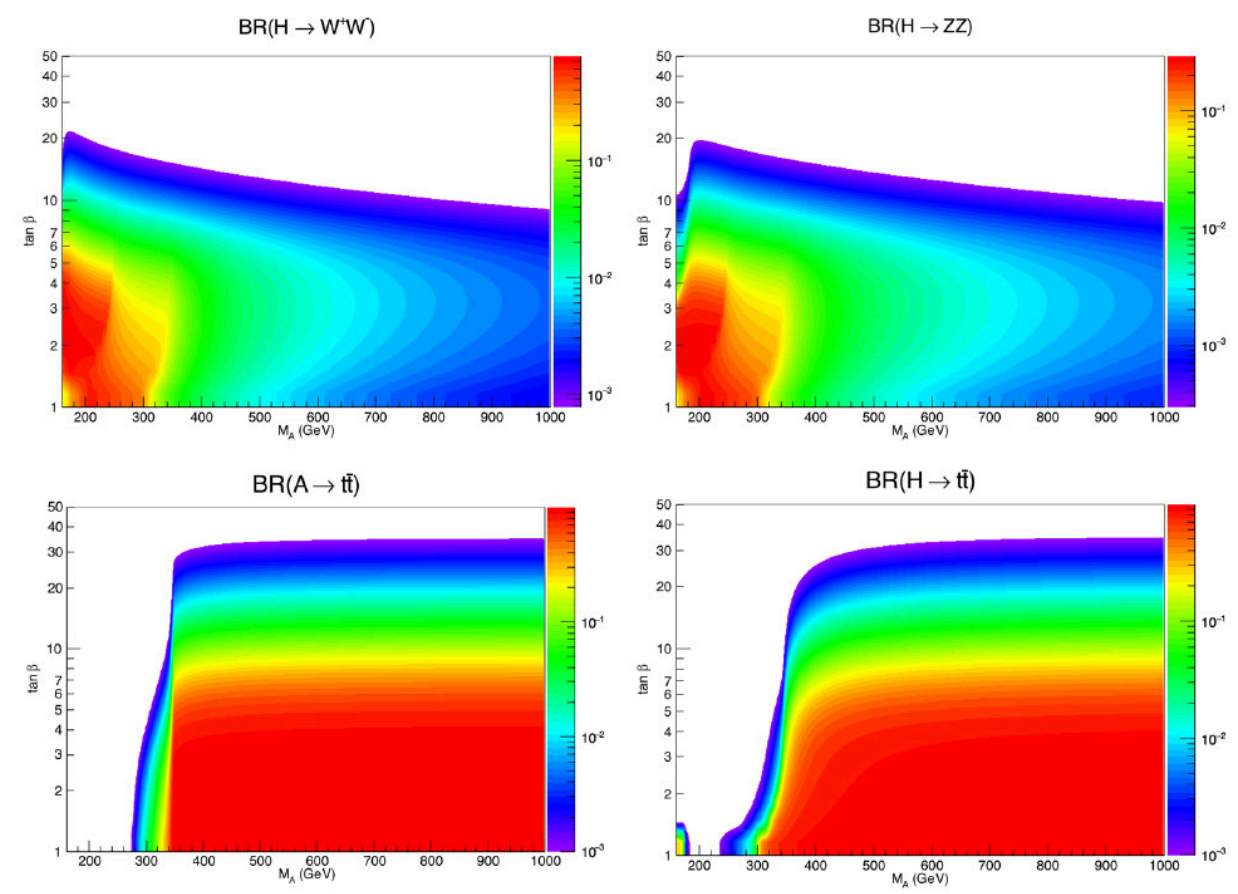

Fig.3. The branching ratios of the neutral Higgs bosons in the $\left[\tan \beta ; M_{A}\right]$ parameter space of the hMSSM model, from [12] 

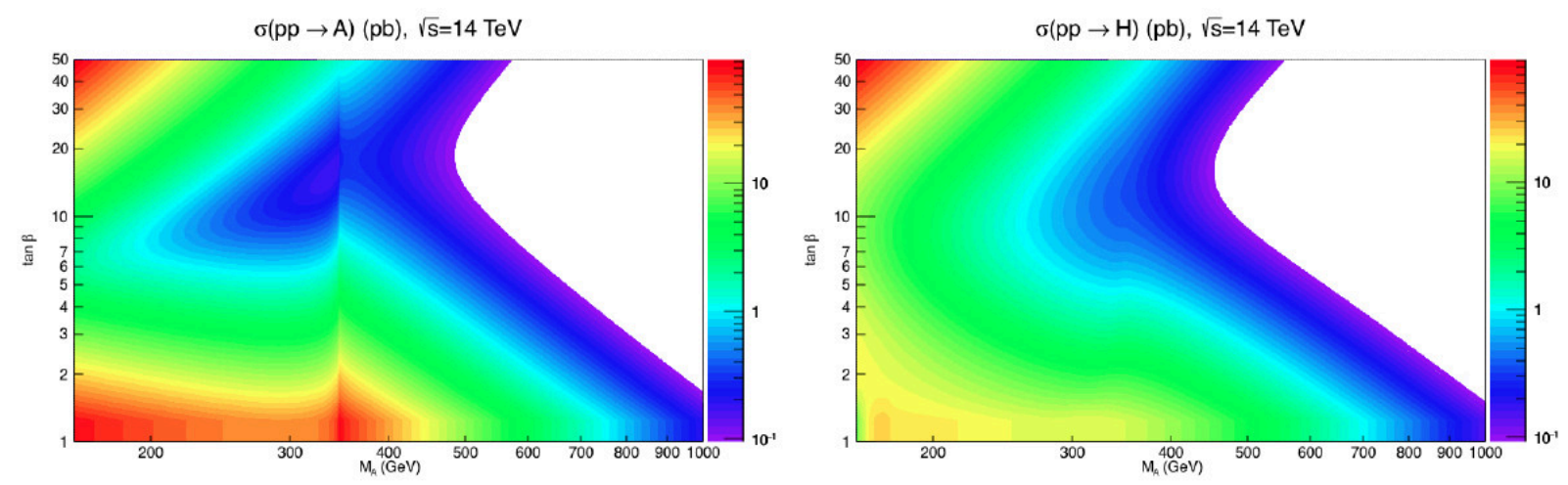

Fig.4. The production cross sections of the Higgs bosons $A$ (left) and $H$ (right) at the LHC with $\sqrt{s}=14$ TeV in the $\left[\tan \beta ; M_{A}\right]$ hMSSM plane, from [12]

3. CALCULATIONS OF THE PRODUCTION CROSS SECTIONS TIMES BRANCHING FRACTIONS FOR HIGGS BOSONS

1) CP-even Higgs boson, $\mathrm{H}$.

Searches for heavy Higgs bosons by Run-2 ATLAS
Collaboration at the LHC in the $H \rightarrow Z Z$ and $H \rightarrow$ $W W$ decay channels are relevant due to the possibility of evidence for new particles beyond the Standard Model. The limits on $\sigma(p p \rightarrow H) \times B R(H \rightarrow Z Z)$ and $\sigma(p p \rightarrow H) \times B R(H \rightarrow W W)$ at $95 \%$ CL from [13] and [14] correspondingly are presented in Fig.5.

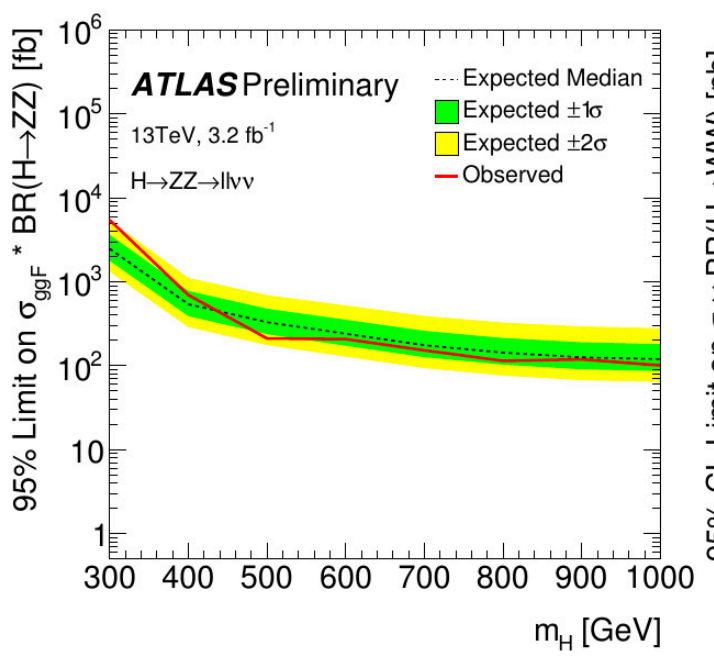

(a)

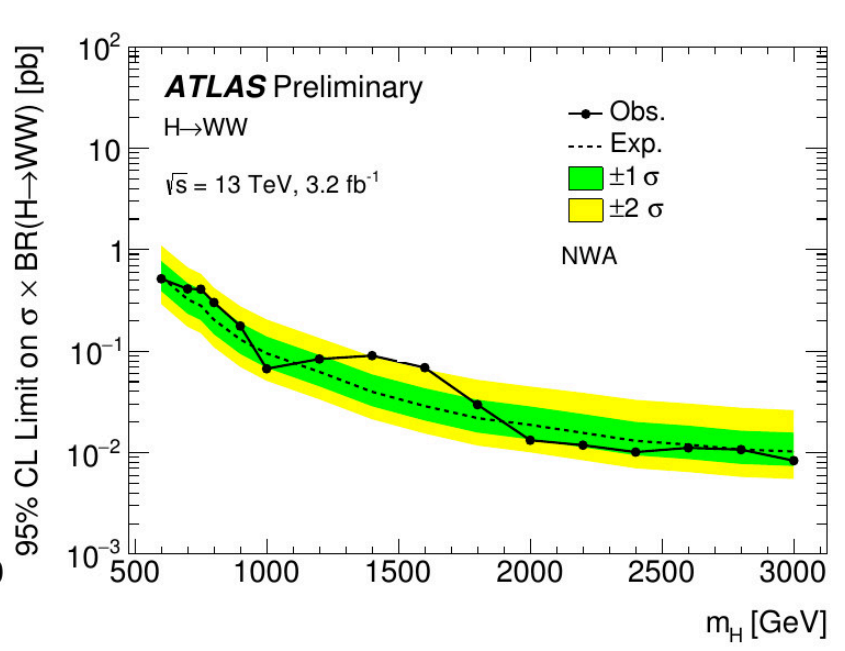

(b)

Fig.5. Limits on $\sigma(p p \rightarrow H) \times B R(H \rightarrow Z Z)$ (a) and $\sigma(p p \rightarrow H) \times B R(H \rightarrow W W)$ (b) via gluon-gluon fusion at $95 \% \mathrm{CL}$

Using the restricted parameter set for $\left[\tan \beta ; \mathrm{M}_{A}\right]$ plane, presented in the previous section and computer programs SusHi [15] and SOFTSUSY4.0 [16], we calculated $\sigma(p p \rightarrow H) \times B R(H \rightarrow Z Z)$ and $\sigma(p p \rightarrow H) \times B R(H \rightarrow W W)$ for $\sqrt{s}=14 \mathrm{TeV}$ at the LHC, presented in Fig.6. 

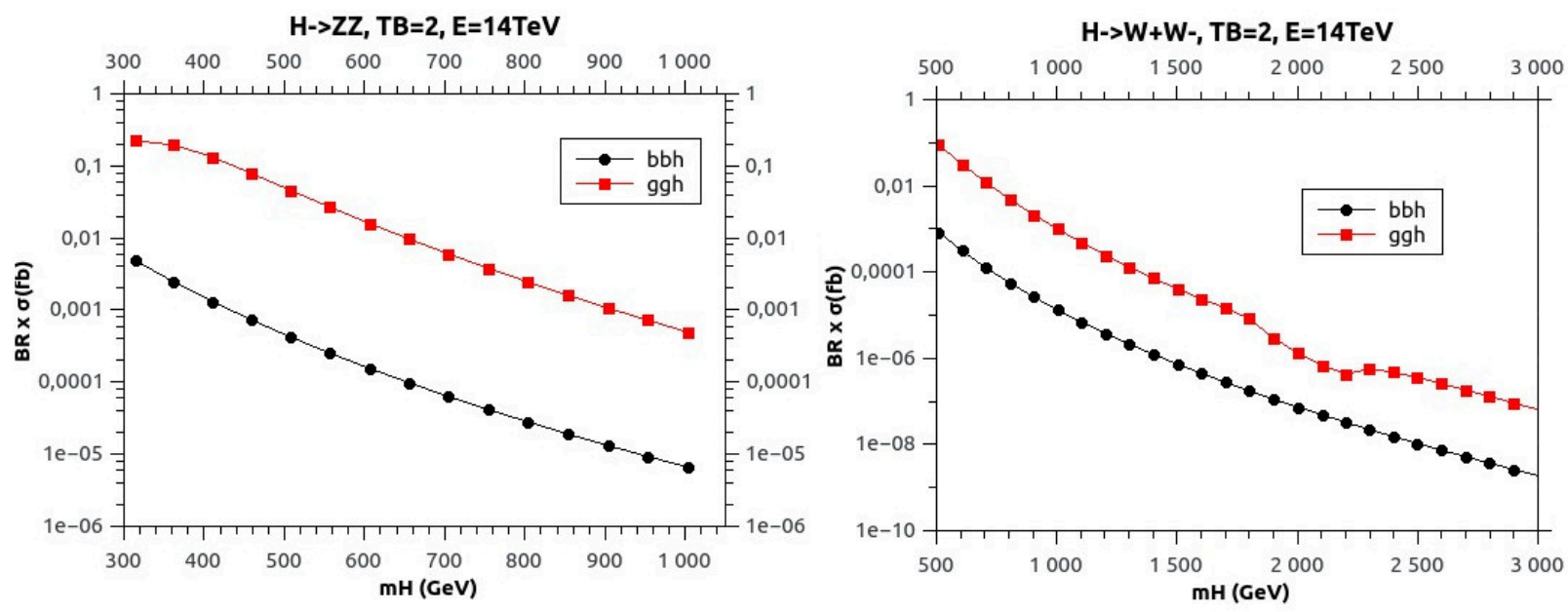

Fig.6. $\sigma(p p \rightarrow H) \times B R(H \rightarrow Z Z)$ (left) and $\sigma(p p \rightarrow H) \times B R(H \rightarrow W W)$ (right) for $\sqrt{s}=14$ TeV at the $L H C$

From Fig.6 we can see the increase in value $\sigma \times B r$ for ggh fusion process compared with bbh fusion process of heavy Higgs boson, $\mathrm{H}$ production. Since the branching ratios for the decays $H \rightarrow b b$ and $H \rightarrow t t$ are significant values according to our calcu- lations with SOFTSUSY4.0 program, we have performed calculations of $\sigma(p p \rightarrow H) \times B R(H \rightarrow t t)$ and $\sigma(p p \rightarrow H) \times B R(H \rightarrow b b)$ for the planned at the LHC energy of $14 \mathrm{TeV}$, presented in Fig.7.
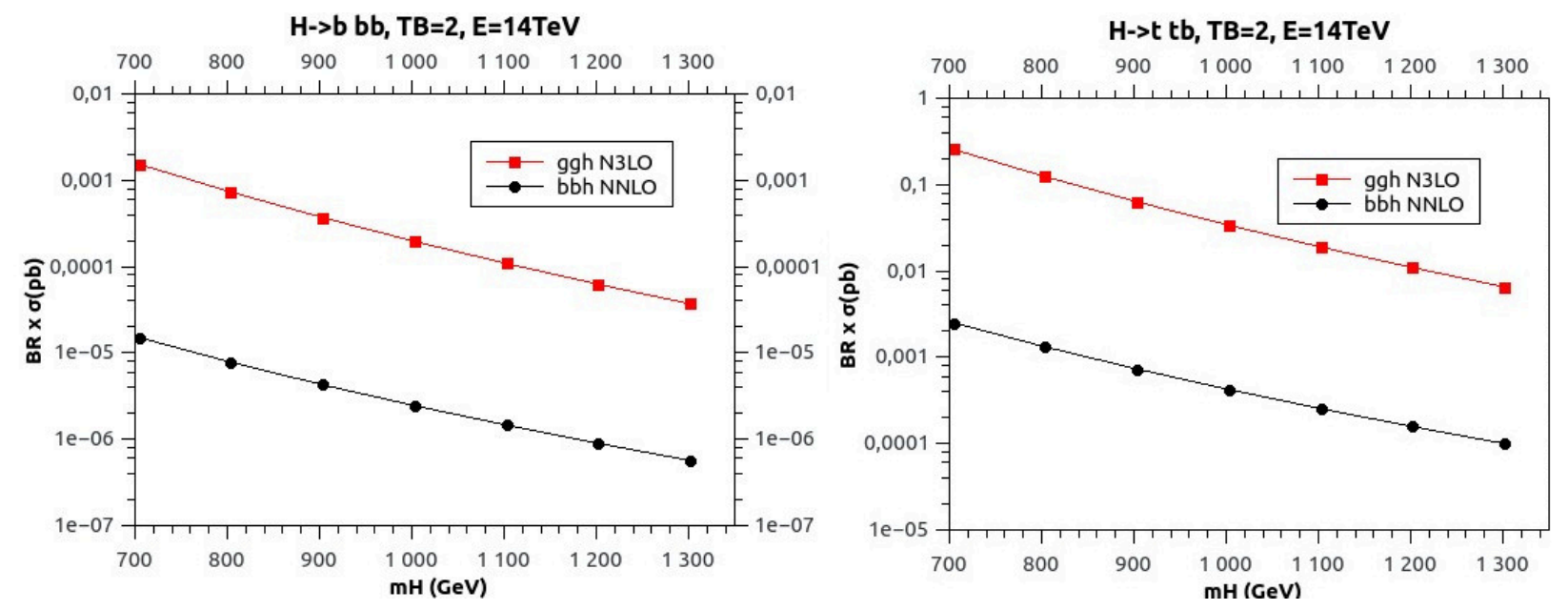

Fig. 7. $\sigma(p p \rightarrow H) \times B R(H \rightarrow b b)$ (left) and $\sigma(p p \rightarrow H) \times B R(H \rightarrow t t)$ (right) for $\sqrt{s}=14$ TeV at the LHC

From the comparison of our calculations, presented above, we can see significant predominance of the values $\sigma \times B r$ for the second variant (see Fig.7) compared to the first one (see Fig.6). It is also important to stress the necessity of N3LO calculations for essential enlargement of the $\sigma \times B r$ value.

2) CP-odd Higgs boson, A.

In this section we have considered the following decay processes of A boson: $A \rightarrow b b$ and $A \rightarrow t t$. The consideration of these processes of A boson decay is connected with the large value of branching ratio, that is represented in Fig.1. As we have calculated the process $A \rightarrow Z h$ in [17] and currently there are no other experimental data, for future experimental searches it was of interest to perform calculations for the two other decay channels from the three maximal. Using the computer programs SOFTSUSY4.0 and SusHi, we have performed the calculations of $\sigma \times \mathrm{Br}$ for CP-odd Higgs boson, A. As the branching ratio for A boson is maximal for the decays $A \rightarrow b b$ and $A \rightarrow t t$ in the selected set of parameters, it was interesting to calculate $\sigma \times B r$ for this both processes over a wide range of boson masses, from $500 \mathrm{GeV}$ to $3450 \mathrm{GeV}$. The results of our calculations are presented in Fig.8. 
$A->b$ bb, TB=2, $E=14 T e V$
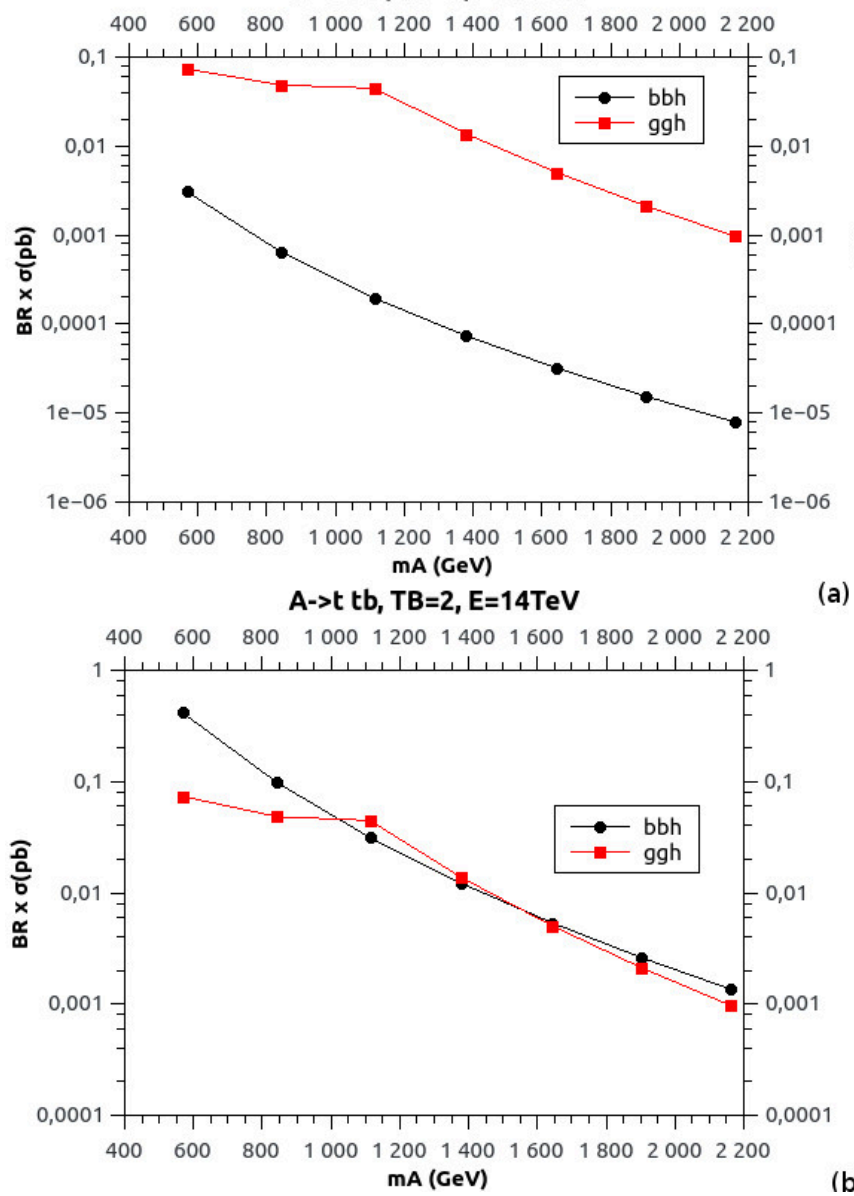

$A->b$ bb, TB=2, $E=14 T e V$

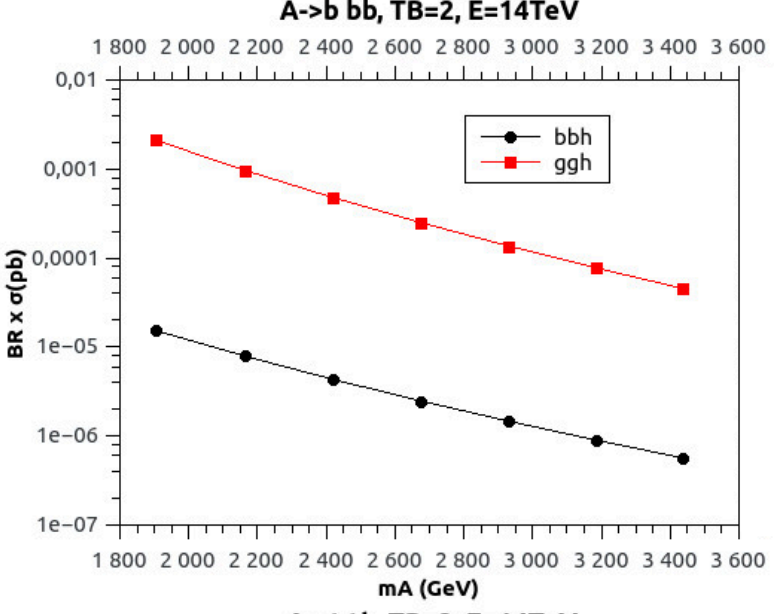

$A->t$ tb, $T B=2, E=14 T e V$

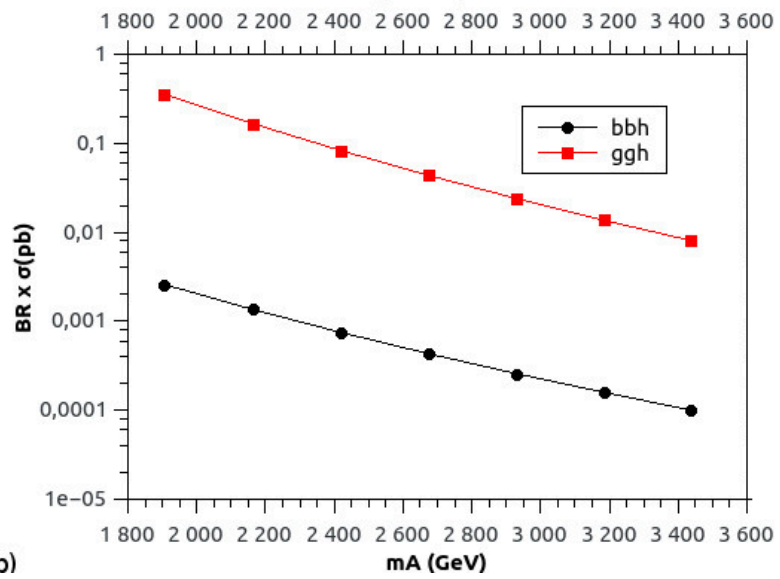

Fig.8. $\sigma(p p \rightarrow A) \times B R(A \rightarrow b b)$ in the mass range $500 \ldots 2200 \mathrm{GeV}$ (left) and $1800 \ldots 3450 \mathrm{GeV}$ (right) (a) and $\sigma(p p \rightarrow A) \times B R(A \rightarrow t t)$ in the mass range $500 \ldots 2200 \mathrm{GeV}$ (left) and $1800 \ldots 3450 \mathrm{GeV}$ (right) (b)

From Fig.8 we can see the predominance of the ggh process of $\mathrm{A}$ boson formation over the bbh one except for the (b) case of $A \rightarrow t t$ process in the mass range of $500 \ldots 2200 \mathrm{GeV}$ with interesting intersection points between bbh and ggh processes. It is also necessary to stress the largest value of $\sigma \times B r$ for the smallest masses, $m_{A}$, what is easily explained in connection with the lower mass of the Higgs boson A.

3) charged Higgs bosons, $\mathrm{H} \pm$.

As is known [18], the production of charged Higgs boson depends on its mass and for $\mathrm{m}_{H^{+}}>\mathrm{m}_{t}, \mathrm{H}^{+}$ production mode is associated with a top quark, as illustrated in Fig.9.

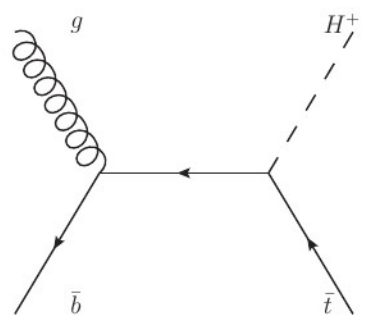

Fig.9. Leading-order Feynman diagram for the production of $\mathrm{H}^{+}$in association with a top quark in five flavor scheme
In Fig.10 are shown the expected and observed limits for the production of $\mathrm{H}^{+} \rightarrow t b$ in association with a top quark, bands for $68 \%$ (in green) and $95 \%$ (in yellow) confidence intervals and the signal prediction in the $\mathrm{m}_{h}^{\text {mod- }}$ benchmark scenario of the MSSM [19].

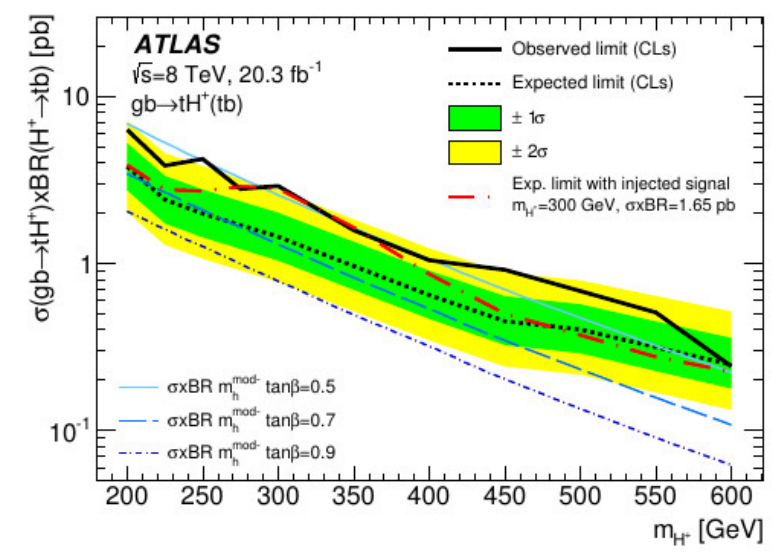

Fig.10. Expected and observed limits for the production of $\mathrm{H}^{+} \rightarrow t b$ in association with a top quark, from [18]

As model points with $0.5 \leq \tan \beta \leq 0.6, \tan \beta \approx$ $0.5, \tan \beta=0.7$ and $\tan \beta=0.9$ are excluded in the $\mathrm{H}^{+}$ mass range of $200 \ldots 600 \mathrm{GeV}$ obtained also in other 
scenarios of MSSM, it would be interesting to do the calculations of $\sigma \times B r$ for $\tan \beta=2$. For the studying of properties of charged Higgs bosons, $\mathrm{H}^{ \pm}$, we have used the set of parameters of MSSM model to calculate the cross-sections of $\mathrm{tH}^{+}$production with the help of the software program PROSPINO [20] with data implemented from the latest computer program SOFTSUSY4.0. The corresponding results for $\sigma\left(p p \rightarrow t H^{+}\right) B R\left(H^{+} \rightarrow t b\right)$, obtained for the parameter set of $\tan \beta=2$ and for the energy of $14 \mathrm{TeV}$ in the mass range of $\mathrm{m} H^{+}=500 \ldots 1200 \mathrm{GeV}$ are presented in Fig.11.

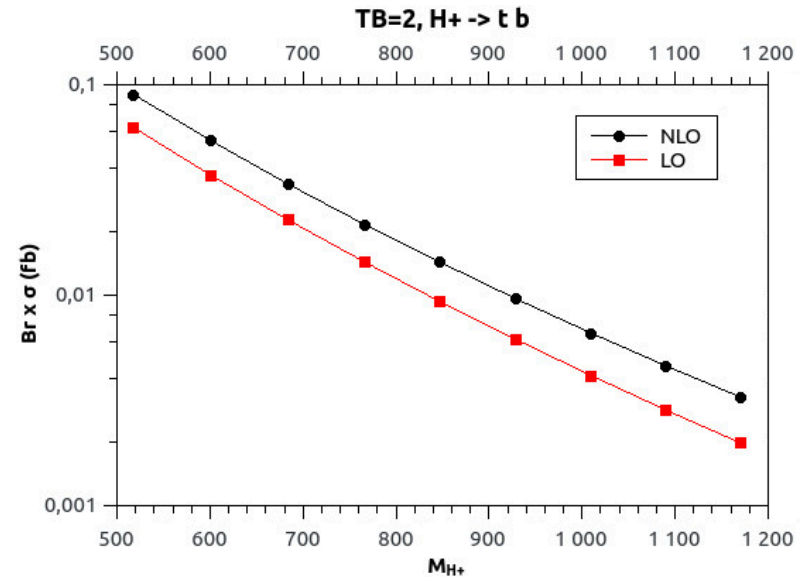

Fig.11. $\sigma\left(p p \rightarrow t H^{+}\right) B R\left(H^{+} \rightarrow t b\right)$ for 14 TeV at the LHC in the mass range of $m_{H^{+}}=500 \ldots 1200 \mathrm{GeV}$

Another most visible decay channel of a charged Higgs boson is $H^{+} \rightarrow \tau \nu$. Its searches in associa- tion with a single top quark were performed by ATLAS Collaboration at the LHC with proton-proton collision at $\sqrt{s}=13 \mathrm{TeV}$ corresponding to an integrated luminosity of $3.2 \mathrm{fb}^{-1}$. The analysis of experimental data leads to $95 \%$ CL upper limits on the $\sigma\left(p p \rightarrow[b] t H^{ \pm}\right) B R\left(H^{ \pm} \rightarrow \tau \nu\right)$, between $1.9 \mathrm{pb}$ and $15 \mathrm{fb}$, for $\mathrm{m}_{H^{+}}=200 \ldots 2000 \mathrm{GeV}$, that is presented in Fig.12.

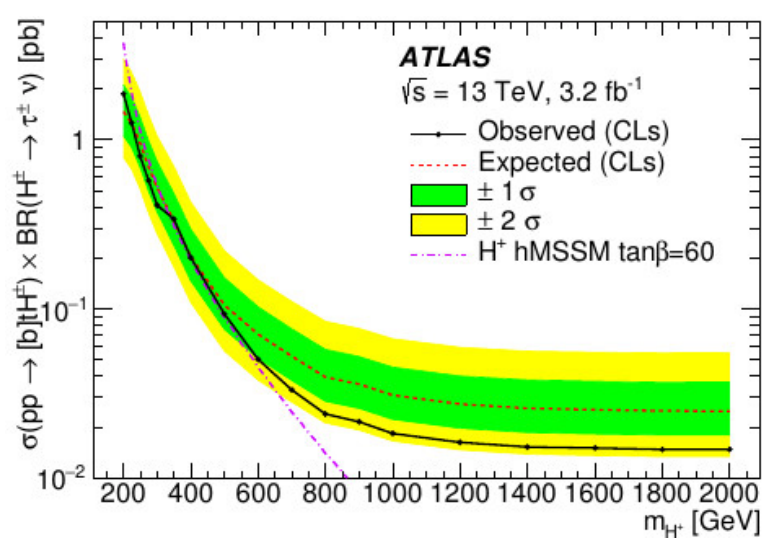

Fig.12. Observed and expected 95\% CL exclusion limits for heavy charged Higgs boson production as a function of $m_{H^{+}}$, from [21]

From these experimental data $\tan \beta=42 \ldots 60$ for $\mathrm{m}_{H^{+}}=200 \mathrm{GeV}$ and $\tan \beta=60$ for the $\mathrm{H}^{+}$mass range from 200 to $340 \mathrm{GeV}$ were excluded. So we have considered two cases of $\tan \beta=2$ and 30 for comparison of the value of $\sigma\left(p p \rightarrow[b] t H^{ \pm}\right) B R\left(H^{ \pm} \rightarrow \tau \nu\right)$ for these two cases, presented in Fig.13.
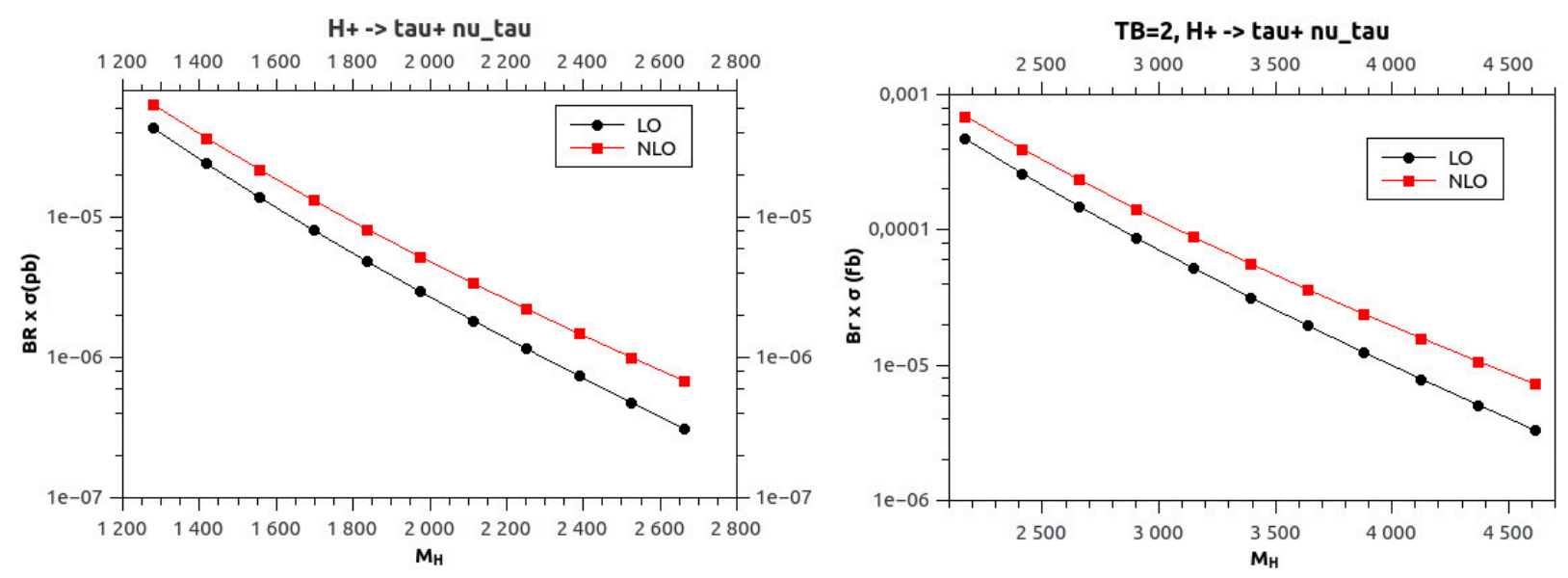

Fig. 13. $\sigma\left(p p \rightarrow[b] t H^{ \pm}\right) B R\left(H^{ \pm} \rightarrow \tau \nu\right)$ for $(a)$ tan $\beta=30$ in the mass range $m_{H}^{+}=1200 \ldots 2650$ GeV and (b) $\tan \beta=2$ in the mass range $m_{H}^{+}=2200 \ldots 4600 \mathrm{GeV}$ with the planned $14 \mathrm{TeV}$ at the LHC

From Fig.13 the predominance in the value of $\sigma\left(p p \rightarrow[b] t H^{ \pm}\right) B R\left(H^{ \pm} \rightarrow \tau \nu\right)$ for the variant (a) is obvious but we can see the larger values of $\sigma\left(p p \rightarrow[b] t H^{ \pm}\right) B R\left(H^{ \pm} \rightarrow \tau \nu\right)$ for $\tan \beta=30$ in the range of the mass intersection of charged Higgs boson, $\mathrm{m}_{H}^{+}=2200 \ldots 2650 \mathrm{GeV}$ for (a) and (b) variants. In addition, it is known that for $m_{H^{+}}>m_{t}$ the dominant decay of $\mathrm{H}^{+}$is $H^{+} \rightarrow t b$, but for large values of $\tan \beta$ is observed a substantial contribution from $H^{+} \rightarrow \tau \nu[21]$. For comparison we calculated $\sigma\left(p p \rightarrow t H^{+}\right) B R\left(H^{+} \rightarrow t b\right)$ for $\tan \beta=30$ for $14 \mathrm{TeV}$ at the LHC, presented in Fig.14.

From the Fig.14 and 13 it can be concluded about the largest values of $\sigma(p p \rightarrow$ $\left.t H^{+}\right) B R\left(H^{+} \rightarrow t b\right)$ in contrast with $\sigma(p p \rightarrow$ $\left.[b] t H^{ \pm}\right) B R\left(H^{ \pm} \rightarrow \tau \nu\right)$ for the same $\tan \beta=30$, but 
the increase of the value $\sigma\left(p p \rightarrow[b] t H^{ \pm}\right) B R\left(H^{ \pm} \rightarrow\right.$ $\tau \nu)$ for the larger $\tan \beta$ was stressed above.

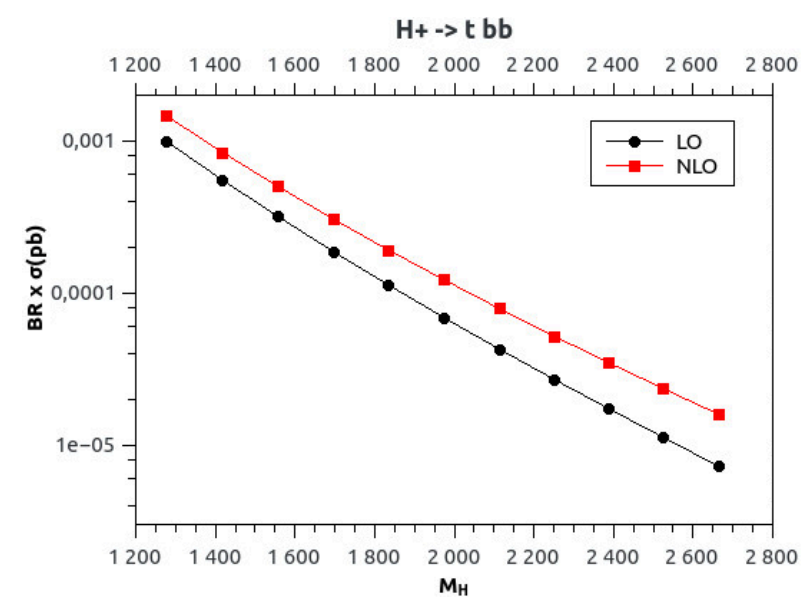

Fig.14. $\sigma\left(p p \rightarrow t H^{+}\right) B R\left(H^{+} \rightarrow t b\right)$ for 14 TeV at the $L H C$ in the mass range of $m_{H}^{+}=1200 \ldots 2650 \mathrm{GeV}$

\section{CONCLUSIONS}

Using the restricted parameter set of the hMSSM model, presented in [5] and [12] for the extended sector of Higgs bosons as well as the latest experimental data on the observed and expected CL exclusion limits for Higgs boson production, performed by ATLAS Collaboration [13], [14], [18], [21] with the help of software programs SOFTSUSY4.0, SusHi and PROSPINO we have calculated $\sigma \times B r$ for CP-even Higgs boson, H, CP-odd Higgs boson, A and charged Higgs bosons, $\mathrm{H}^{ \pm}$. From our calculations we can conclude about the large values of the $\sigma \times B r$ at small $\tan \beta=2$ for chosen decay channels of Higgs bosons for the energy at the LHC of $14 \mathrm{TeV}$. But for the charged Higgs boson are obtained another results, that are connected with larger values of $\tan \beta$.

\section{References}

1. Yuri Shirman. TASI 2008 Lectures: Introduction to Supersymmetry and Supersymmetry Breaking // arXiv:0907.0039v1 [hep-ph] 1 Jul 2009.

2. CMS Collaboration. Search for supersymmetry in multijet events with missing transverse momentum in proton-proton collisions at 13 TeV // CMS-SUS-16-033, CERN-EP-2017-072, arXiv:1704.07781 [hep-ex].

3. CMS Collaboration. SUSY searches in the electroweak sector// CERN Courier. 2017, v.57, N5, p.12.

4. Stephen P. Martin. A Supersymmetry Primer// arXiv: hep-ph/9709356.

5. LHC Higgs Cross Section Working Group/ D. de Florian, C. Grojean, F. Maltoni,
C. Mariotti, A. Nikitenko, M. Pieri, P. Savard, M. Schumacher, R. Tanaka (Eds.). Handbook of LHC Higgs Cross Sections: 4. Deciphering the nature of the Higgs sector // CERN Yellow Reports: Monographs. CERN, Geneva, 2017, v.2, 869p., CERN-2017-002-M.

6. A. Djouadi and J. Quevillon. The MSSM Higgs sector at a high MSUSY: reopening the low $\tan \beta$ regime and heavy Higgs searches // JHEP. 2013, v.10, p.028, arXiv:1304.1787 [hep-ph].

7. E. Bagnaschi et al. Benchmark scenarios for low $\tan \beta$ in the MSSM // LHCHXSWG-2015-002, 2015 .

8. The ATLAS collaboration. Search for neutral Higgs bosons of the minimal supersymmetric standard model in pp collisions at $\mathrm{s}=8 \mathrm{TeV}$ with the ATLAS detector // JHEP 2014, v.11, p.056; arXiv:1409.6064 [hep-ex].

9. The CMS collaboration. Search for neutral MSSM Higgs bosons decaying to a pair of tau leptons in pp collisions // JHEP 2014, v.10, p.160, arXiv:1408.3316 [hep-ex].

10. M. Carena, S. Heinemeyer, C. Wagner and G. Weiglein. Suggestions for Benchmark Scenarios for MSSM Higgs Boson Searches at Hadron Colliders // Eur. Phys. J. 2003, v.C26, p.601607.

11. A. Djouadi, J. Kalinowski and M.Spira. HDECAY: a Program for Higgs Boson Decays in the Standard Model and its Supersymmetric Extension // Comput. Phys. Commun. 1998, v.108, p.56-74;

A. Djouadi, M. Muhlleitner and M. Spira. Decays of supersymmetric particles: The Program SUSY-HIT (SUspect-SdecaY-HdecayInTerface) // Acta. Phys. Polon. 2007, v.B38, p.635-644.

12. A. Djouadi et al. Fully covering the MSSM Higgs sector at the LHC // arXiv:1502.05653v2 [hepph] 22 Jun 2015.

13. The ATLAS Collaboration. Search for highmass resonances decaying into a $\mathrm{Z}$ boson pair in the ll $\nu \nu$ final state in pp collisions at $\mathrm{s}=13 \mathrm{TeV}$ with the ATLAS detector // ATLAS-CONF2016-012.

14. The ATLAS Collaboration. Search for a highmass Higgs boson decaying to a pair of $\mathrm{W}$ bosons in pp collisions at $\mathrm{s}=13 \mathrm{TeV}$ with the ATLAS detector// ATLAS-CONF-2016-021.

15. SusHi can be downloaded from: http: // sushi.hepforge.org/.

16. B. C. Allanach, T. Cridge. The Calculation of Sparticle and Higgs Decays in the Minimal and Next-to-Minimal Supersymmetric Standard 
Models: SOFTSUSY4.0 // arXiv:1703.09717 [hep-ph].

17. T.V. Obikhod, E. A. Petrenko. The study of the extended Higgs boson sector within 2HDM model // arXiv:1703.09776 [hep-ph].

18. ATLAS Collaboration. Search for charged Higgs bosons in the $\mathrm{H}^{ \pm} \rightarrow t b$ decay channel in pp collisions at $\mathrm{s}=8 \mathrm{TeV}$ using the ATLAS detector // JHEP. 2016, v.03, p.127.

19. M. Carena, S. Heinemeyer, O. Stal, C.E.M. Wagner and G. Weiglein. MSSM Higgs Boson Searches at the LHC: Benchmark
Scenarios after the Discovery of a Higgs-like Particle // Eur. Phys. J. 2013, v.C 73, 2552, arXiv:1302.7033.

20. W. Beenakker, R. Hoepker, M. Spira. PROSPINO: A Program for the Production of Supersymmetric Particles in Next-to-leading Order QCD // arXiv:hep-ph/9611232v1.

21. The ATLAS Collaboration. Search for charged Higgs bosons produced in association with a top quark and decaying via $\mathrm{H} \rightarrow \tau \nu$ using pp collision data recorded at $\mathrm{s}=13 \mathrm{TeV}$ by the ATLAS detector // CERN-PH-2016-056.

\section{ИЗУЧЕНИЕ СВОЙСТВ РАСШИРЕННОГО СЕКТОРА БОЗОНА ХИГГСА В РАMКАХ hMSSM-МОДЕЛИ}

\section{Т. В. Обиход, Е. А. Петренко}

Используя последние экспериментальные данные, полученные ATLAS коллаборацией, в рамках Минимальной суперсимметричной стандартной модели, мы представили расчеты по сечениям умноженным на ширины распадов, $\sigma \times \mathrm{Br}$, как функции массы СР-четного $\mathrm{H}$ бозона Хиггса, $\mathrm{CP}$-нечетного А бозона Хиггса и заряженного, $H^{ \pm}$бозона Хиггса. Использование ограниченного набора параметров, полученных из hMSSM + HDECAY и "low-tb-high"сценариев, с помощью компьютерных программ SOFTSUSY, Prospino и SusHi, дало возможность получить большие значения $\sigma \times \mathrm{Br}$ для $\mathrm{A}$ и $\mathrm{H}$ бозонов при $\tan \beta$ $=2$ для запланированных $14 \mathrm{T \ni}$ В на LHC и большое значение $\sigma \times \mathrm{Br}$ при $\tan \beta=30$ для заряженного бозона Хиггса. Полученные результаты представляют собой интерес для эксперимента, поскольку они связаны с экспериментальными поисками новой физики за пределами Стандартной модели на LHC.

\section{ВИВЧЕННЯ ВЛАСТИВОСТЕЙ РОЗШИРЕНОГО СЕКТОРА БОЗОНА ХІГГСА В PAMКАХ hMSSM-МОДЕЛI \\ Т. В. Обіход, Є. О. Петренко}

Використовуючи останні експериментальні дані, отримані ATLAS колаборацією, у рамках Мінімальної суперсиметричної стандартної моделі, ми представили розрахунки по перерізам утворення помноженим на ширини розпадів, $\sigma \times \mathrm{Br}$, як функції маси СР-парного Н бозона Хіггса, СР-непарного А бозона Хіггса і зарядженого $H^{ \pm}$бозона Хіггса. Використання обмеженого набору параметрів, отриманого з $\mathrm{hMSSM}+$ HDECAY i "low-tb-high"сценаріїв, за допомогою комп'ютерних програм SOFTSUSY, Prospino i SusHi, дало можливість отримати великі значення $\sigma \times \mathrm{Br}$ задля $\mathrm{A}$ i $\mathrm{H}$ бозонів при $\tan \beta=2$ для запланованих $14 \mathrm{TeB}$ на LHC і велике значення $\sigma \times \mathrm{Br}$ при $\tan \beta=30$ для зарядженого бозона Хіггса. Отримані результати $\epsilon$ важливими для експеримента, оскільки вони пов'язані із експериментальними пошуками нової фізики за межами Стандартної моделі на LHC. 